\title{
¿Qué opinan los médicos penitenciarios sobre el control de la infección por el VIH en las prisiones españolas? Resultados del Estudio del Grupo de Trabajo en Enfermedades Infecciosas de la Sociedad Española de Sanidad Penitenciaria (GEISESP)
}

\author{
P Saiz De la Hoya ${ }^{1}$, A Marco $^{2}$, J García-Guerrero \\ ${ }^{1}$ Centro Penitenciario Alicante 1 (Fontcalent) \\ ${ }^{2}$ Centro Penitenciario Barcelona Hombres; \\ ${ }^{3}$ Centro Penitenciario Castellón
}

\section{RESUMEN}

Se presentan los resultados del estudio realizado por el Grupo de Enfermedades Infecciosas de la Sociedad Española de Sanidad Penitenciaria (GEISESP) sobre la opinión de los facultativos penitenciarios en cuanto a la conveniencia o no de realizar el control de la infección por el VIH en el medio carcelario y sobre las dificultades, esfuerzos, necesidades y responsabilidades que esta actividad puede suponer. Este estudio se efectuó en el año 2007 utilizando una encuesta diseñada ad hoc remitida a todos los facultativos de 73 centros penitenciarios españoles. Se recibieron 116 cuestionarios válidos, estimándose que los datos obtenidos podían aplicarse al total de la población con un nivel de confianza del $95 \%$ y un margen de error del $8 \%$. La mitad de los médicos refirieron prescribir tratamientos antirretrovirales, aunque sólo el 10\% decían prescribir combinaciones de rescate. Es de destacar también que sólo la mitad de los médicos se consideran formados adecuadamente para atender pacientes infectados por el VIH, aunque la mayoría afirma haber hecho formación específica de postgrado en esta materia. En general, los médicos consideran que la calidad de la asistencia recibida por los infectados por el VIH en prisión es buena.

Palabras clave: Prisiones, VIH, Terapia Antirretroviral Altamente Activa.

\section{WHAT DO PRISON DOCTORS THINK ABOUT HIV INFECTION CONTROL IN SPANISH PRISONS? RESULTS OF THE STUDY OF THE SPANISH SOCIETY OF PRISON HEALTH WORKING GROUP ON INFECTIOUS DISEASES (GEISESP)}

\begin{abstract}
We present the results of the study carried out by The Spanish Society of Penitentiary Health Working Group on Infectious Diseases (GEISESP) on the opinion of prison doctors concerning the suitability of undertaking or not undertaking controls of HIV infection in prisons and about the difficulties, efforts, needs and responsibilities that this activity may involve. This study was carried out in 2007 using an ad hoc survey designed and sent to doctors at 73 prisons in Spain. 116 valid questionnaires were received. It was considered that the data received could be applied to the whole population with a level of trust of $95 \%$ and an $8 \%$ margin of error. Half the doctors referred to prescribing antiretroviral treatments, though only $10 \%$ admitted prescribing rescue combinations. It should be noted that only of half the doctors consider themselves to be appropriately prepared to deal with patients infected by the HIV virus, though half of them admit to having received spe-
\end{abstract}


cific postgraduate training on this subject. Generally speaking, doctors consider that the quality of the health care received by the HIV-infected population is quite good.

Key words: Prisons, HIV, Antiretroviral Therapy, Highly Active .

Texto recibido: diciembre 2008

Texto aceptado: mayo 2009

\section{INTRODUCCION}

La población reclusa española, ya sea dependiente de la Administración del Estado o del Gobierno de Cataluña, ha aumentado enormemente en los últimos 30 años. Además, en este periodo de tiempo, también ha presentado importantes modificaciones cualitativas, sobre todo a partir de tres hechos cruciales. El primero, producido por los cambios legislativos ocurridos tras la reinstauración de la democracia, que modificaron o eliminaron el carácter punitivo que hasta entonces presentaban algunas conductas y, consecuentemente, modificaron el perfil sociológico de una buena parte de los ingresados en prisión. El segundo, de enorme relevancia, relacionado con el consumo de heroína en España, prácticamente inexistente antes de 1977, pero que presentó una rápida expansión entre 1979 y 1982 y alcanzó su cenit epidémico entre 1983 y 1986 ${ }^{1}$. Estos miles de consumidores de opiáceos se caracterizaron por realizar repetidas actividades delictivas, necesarias en muchos casos para mantener su adicción ${ }^{2}$, lo que explica el encarcelamiento habitual de este tipo de población: joven, con graves carencias en materia de salud y con un código de valores modificado por su dependencia a las drogas. Finalmente, el tercer hecho crucial, ocurrido más recientemente y que se prolonga hasta la actualidad, guarda relación con el fenómeno migratorio, que ha ocasionado una creciente población presa extranjera, que en la actualidad supone aproximadamente un tercio del total de reclusos en España ${ }^{3}$ (aunque en algunas autonomías como Cataluña asciende hasta un $41 \%{ }^{4}$ ). Esta población, habitualmente producto de la emigración secundaria a las malas condiciones socioeconómicas de sus países de origen, suele diferenciarse de los presos españoles por un menor consumo endovenoso de drogas, una peor integración social y, en ocasiones, una mayor profesionalización delictiva ${ }^{5}$. Sanitariamente, pueden ser portadores de procesos transmisibles poco frecuentes en nuestro medio y presentan una baja inmunidad frente a algunas enfermedades propias de nuestro entorno.

La aplicación de programas de reducción de daños, el descenso del número de heroinómanos y un cambio en el patrón de consumo a partir de 1990 ha supuesto una reducción ${ }^{6}$ de los usuarios de drogas intravenosas $(\mathrm{UDI})^{7,8}$ que ingresan en prisión y, secundariamente, una disminución de la prevalencia de infección por el VIH, que ha pasado en la población internada del $25 \%$, a finales de los años 80 , al $15 \%$ en Cataluña y al 9-12\% en el resto del Estado-11 en la actualidad. A pesar de ello, esta prevalencia es la más alta de los países de la Unión Europea y supone - dada la complejidad y coste económico del control sanitario de estos pacientes - un reto enormemente importante para la Administración Pública. En algunas revisiones ${ }^{12,13}$ se han analizado las ventajas y desventajas de que el control de los infectados por el VIH se efectúe en el propio centro penitenciario, pero sin embargo no se conoce lo que opinan los profesionales sobre la conveniencia o no de realizar este control en el medio carcelario y sobre las dificultades, esfuerzos, necesidades y responsabilidades que esta actividad puede suponer. Con la pretensión de conocer estas opiniones, se presenta el estudio realizado por el Grupo de Enfermedades Infecciosas de la Sociedad Española de Sanidad Penitenciaria (GEISESP).

\section{MATERIAL Y MÉTODOS}

Se remitió en mayo de 2007 una encuesta diseñada ad hoc a todos los facultativos (más de 400) de los 90 centros penitenciarios españoles, excluyendo los centros de inserción social y los psiquiátricos. Se respondieron 116 cuestionarios, estimándose que los datos obtenidos podían aplicarse al total de la población con un nivel de confianza del $95 \%$ y un margen de error del $8 \%$.

La encuesta se subdividía en cinco aparatados. El primero, definía las características de la población encuestada; el segundo, intentaba averiguar las actitudes profesionales de los encuestados respecto a los pacientes infectados por el VIH; el tercero, pretendía conocer la opinión del profesional sobre la atención recibida por estos pacientes; el cuarto, intentaba averiguar la dotación de medios sanitarios, materiales y humanos, disponibles en el centro de trabajo; y el 
Rev Esp Sanid Penit 2009; 11: 42-48

P Saiz De la Hoya, A Marco, J García-Guerrero. ¿Qué opinan los médicos penitenciarios sobre el control de la infección por el VIH en las prisiones españolas?

quinto, quería conocer cuáles, a criterio de los encuestados, eran los principales problemas que la infección generaba en la prisión, así como las ventajas y desventajas de realizar el control y tratamiento en el medio penitenciario.

Los datos cuantitativos observados en las encuestas son siempre subjetivos y han sido emitidos en base a las apreciaciones personales y experiencia de los profesionales que respondieron al cuestionario.

\section{RESULTADOS}

\subsection{Características de la población encuestada}

La mayoría de los facultativos que contestaron fueron varones $(62,9 \%)$, mayores de 40 años $(85,3 \%)$, médicos generales $(67,2 \%)$, y con experiencia mayor de 10 años (Tabla 1$)$.

\subsection{Características de la atención prestada al paciente infectado por el VIH}

La mayoría de los facultativos ( $\mathrm{n}=113 ; 97,4 \%)$ refieren atender a pacientes infectados por el VIH. Dicen hacerlo habitualmente el 51,7\% y frecuentemente el $45,7 \%$. El 1,7\% manifiesta que raramente atiende a este tipo de pacientes y sólo el $0,9 \%$ dice no hacerlo nunca. Además, el 56,9\% lleva atendiendo a pacientes infectados por el VIH más de 15 años. El número de pacientes infectados que atiende cada médico es menor de 50 en el $62,9 \%$ de los casos, independientemente del número de internos por centro que haya en su centro. No obstante, el $34,5 \%$ atiende a más de 50 pacientes y el 4,3\% atiende a más de 100 pacientes.

Cuando se concreta el grado de atención, casi la mitad $(49,1 \%)$ realizan exclusivamente control básico del paciente (definido éste como una actuación mé-

\begin{tabular}{|c|c|c|}
\hline & & n \% \\
\hline \multirow{2}{*}{ Sexo } & Hombre & $73(62,9)$ \\
\hline & Mujer & $33(37,1$ \\
\hline \multirow{3}{*}{ Edad } & $30-40$ años & $13(11,2)$ \\
\hline & $41-50$ años & $86(74,1)$ \\
\hline & $>50$ años & $17(14,7)$ \\
\hline \multirow{5}{*}{ Distribución Geográfica } & Andalucía/Extremadura & $29(25)$ \\
\hline & Castillas/Madrid & $31(26,7)$ \\
\hline & Galicia/Asturias/País Vasco/Rioja/Aragón & $20(17,2)$ \\
\hline & Islas Baleares/Canarias & $9(7,8)$ \\
\hline & Cataluña/Valencia/Murcia & $27(23,2)$ \\
\hline \multirow{3}{*}{ Especialidad } & Médico General & $78(67,2)$ \\
\hline & Medicina de Familia & $32(27,6)$ \\
\hline & Otras & $6(5,2)$ \\
\hline \multirow{2}{*}{ Experiencia profesional } & $>15$ años & $100(86,2)$ \\
\hline & $<5$ años & $4(3,4)$ \\
\hline \multirow{2}{*}{ Experiencia en prisiones } & $\geq 10$ años & $74(63,8)$ \\
\hline & $<5$ años & $17(14,7)$ \\
\hline \multirow{3}{*}{ Tipo de centro donde trabaja } & Penados & $11(9,5)$ \\
\hline & Preventivos & $32(27,6)$ \\
\hline & Mixtos & $73(62,9)$ \\
\hline \multirow{2}{*}{ Tamaño del centro donde trabaja } & $<1000$ internos & $50(43,1)$ \\
\hline & $>1000$ internos & $66(56,9)$ \\
\hline
\end{tabular}

Tabla 1: Características de la población encuestada $(n=116)$. 
dica circunscrita a la solicitar las determinaciones analíticas y realizar los controles clínicos programados periódicamente). Es de resaltar que prescriben tratamiento antirretroviral el 46,5\% de los médicos, pero sólo el $11,2 \%$ realiza esta prescripción en rescates o en líneas terapéuticas avanzadas. Se observa una tendencia a mayor prescripción de antirretrovirales cuanto mayor es la experiencia profesional en el ámbito penitenciario. De la misma manera, la existencia de alguna especialización conlleva mayor grado de prescripción $(38,5 \%$ de los médicos generales versus $65,7 \%$ de los médicos de familia).

La mitad de los encuestados $(54,3 \%)$ considera que el control de estos pacientes debería estar incentivado económicamente, así como que también debe estarlo otro tipo de actividades asistenciales más especializadas. El 31,9\% refiere, además, que aumentaría su nivel de intervención si se mejorase su formación. Sin embargo, muy pocos médicos $(2,6 \%)$ decla- ran no estar formados para atender a estos pacientes, aunque el $56 \%$ no se consideran del todo formados. Debe destacarse que un alto porcentaje $(87,9 \%)$ afirma haber realizado alguna formación postgrado específica sobre la infección por el VIH. El 62,9\% de los encuestados afirman asistir a más de una reunión científica al año sobre VIH. Se declaran "en parte especializados" para atender a estos pacientes el 58,6\% de los encuestados y "especializados" el 19,8\%. Son los médicos que llevan menos tiempo ejerciendo los que consideran que no están especializados (el 100\% de los que ejercen menos de 5 años, y el 66,7\% de entre 5 y 10 años). No existen diferencias en dependencia de la especialidad, exceptuando a los internistas que se consideran todos especializados.

El 38,8\% de los encuestados opina que esta función conlleva responsabilidades que no debe asumir.

En cuanto a la prescripción farmacológica en otras enfermedades infecciosas, se observa:

\% Médicos que realizan el control del VIH en su centro

$\%$ de médicos que prescriben antirretrovirales

Calidad de la asistencia sanitaria que se da en su centro

\begin{tabular}{lc} 
& n (\%) \\
\hline Ninguno & $1(09)$ \\
\hline$<50 \%$ & $16(13,8)$ \\
\hline $50 \%$ & $36(31)$ \\
\hline Todos & $60(51)$ \\
\hline No sabe no contesta & $3(2,6)$ \\
\hline Ninguno & $22(19)$ \\
\hline$<50 \%$ & $41(55.3)$ \\
\hline$>50 \%$ & $34(29,3)$ \\
\hline Todos & $18(15,5)$ \\
\hline No sabe no contesta & $1(0.9)$ \\
\hline Mala & $1(0,9)$ \\
\hline Regular & $13(11,2)$ \\
\hline Suficiente & $20(17.2)$ \\
\hline Buena & $77(66.4)$ \\
\hline Dependiendo del problema & $5(4,3)$ \\
\hline No sabe no contesta & 0 \\
\hline Mala & $1(0.9)$ \\
\hline Regular & $14(12.1)$ \\
\hline Suficiente & $18(15.5)$ \\
\hline Buena & $83(71,5)$ \\
\hline Dependiendo del problema & 0 \\
\hline No sabe no contesta & 0 \\
\hline
\end{tabular}

Se define control médico del paciente VIH como: Actuación médica reducida a la indicación de las determinaciones analiticas periódicas que estos pacientes requieren más los controles clínicos periódicos necesarios programados.

Tabla 2: Características de atención prestada al paciente infectado por el VIH 
Rev Esp Sanid Penit 2009; 11: 42-48

P Saiz De la Hoya, A Marco, J García-Guerrero. ¿Qué opinan los médicos penitenciarios sobre el control de la infección por el VIH en las prisiones españolas?

Que el 91,4\% de los profesionales dice prescribir tratamiento para la tuberculosis o la infección tuberculosa latente.

Que el 47,4\% de los encuestados refiere prescribir tratamiento para la hepatitis crónica por VHC (hay que recordar que el $46,5 \%$ lo hacían en el caso del VIH).

\subsection{Características de la calidad de la atención que se presta al paciente infectado por el VIH en prisión}

La mayoría de los médicos realizan los controles analíticos a la población infectada por el VIH, pero menos del $50 \%$ prescriben tratamiento antirretroviral. La mayoría piensa que la calidad asistencial global $(66,4 \%)$ que se da en su centro es buena, y también es buena $(71,5 \%)$ la que se da al paciente VIH (Tabla 2).

\subsection{Sobre los medios humanos y materiales disponibles para la atención del paciente infectado por el VIH}

A la mayoría de los centros no acude un consultor externo especialista en VIH (69\%). En prácticamente todos los centros se pueden solicitar cargas virales VIH y subpoblacines linfocitarias, pero en me-

\begin{tabular}{|c|c|c|}
\hline & & n (\%) \\
\hline \multirow{2}{*}{ Acude a su Centro un especialista externo en VIH } & SÍ & $36(31)$ \\
\hline & $\overline{\mathrm{NO}}$ & $80(69)$ \\
\hline \multirow{2}{*}{$\begin{array}{l}\text { Si acude, ¿es el único profesional que atiende a la } \\
\text { población VIH }\end{array}$} & SÍ & $3(8,3)$ \\
\hline & $\overline{\mathrm{NO}}$ & $33(91,7)$ \\
\hline
\end{tabular}

Las siguientes pruebas, ¿se realizan en su centro a petición de $\mathrm{Vd}$ ?

Determinación de Carga Viral VIH

SÍ $114(98.3)$

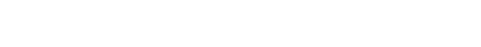

Determinación de Linfocitos CD4

NO $2(17)$

$\mathrm{NS} / \mathrm{NC}$

Sí $114(98.3)$

\begin{tabular}{|c|c|c|}
\hline \multirow[t]{2}{*}{ Determinación de Linfocitos CD4 } & $\mathrm{NO}$ & $1(0.9)$ \\
\hline & $\mathrm{NS} / \mathrm{NC}$ & $1(0.9)$ \\
\hline \multirow{3}{*}{ Niveles terapéuticos de FARV } & Sí & $39(33.6)$ \\
\hline & $\mathrm{NO}$ & $63(54.3)$ \\
\hline & $\mathrm{NS} / \mathrm{NC}$ & $14(12.1)$ \\
\hline \multirow{3}{*}{ Fibroscan } & SÍ & $15(12.9)$ \\
\hline & $\underline{\mathrm{NO}}$ & $61(52.6)$ \\
\hline & $\mathrm{NS} / \mathrm{NC}$ & $40(34.5)$ \\
\hline \multirow{4}{*}{ Test de resistencias a FARV } & Sí y en cualquier circunstancia & $(48,3)$ \\
\hline & Sí en determinadas circunstancias & $(22.4)$ \\
\hline & No tiene acceso & $(27.6)$ \\
\hline & $\mathrm{NS} / \mathrm{NC}$ & $(1.7)$ \\
\hline \multirow{2}{*}{ Es posible hacer TDO en el tratamiento de los pacientes VIH } & Sí & $88(75.9)$ \\
\hline & $\mathrm{NO}$ & $28(24,1)$ \\
\hline \multirow{3}{*}{ El TDO se realiza correctamente } & Sí & $(72,4)$ \\
\hline & $\mathrm{NO}$ & $(23.3)$ \\
\hline & $\overline{\mathrm{NS} / \mathrm{NC}}$ & $(4.3)$ \\
\hline
\end{tabular}

FARV: Fármacos antirretrovirales; TDO: Terapia directamente observada

Tabla 3: Características de los medios, humanos y materiales, disponibles en los centros penitenciarios 
nor proporción se puede solicitar la realización de un fibroscan, tests de resistencias a fármacos antirretrovirales o niveles plasmáticos de fármacos (Tabla 3).

\subsection{Ventajas, desventajas e inconvenientes del medio penitenciario como escenario de control de la infección VIH}

Los médicos consideran que los problemas más habituales en el control de la infección por el VIH en prisión guardan relación sobre todo con el tratamiento $(39,1 \%$ de los casos): adherencia, fracaso, terapia directamente observada, efectos adversos, etc. Y en menor porcentaje $(23,5 \%)$ son problemas derivados de la propia enfermedad o relacionados con la coinfección con el VHC. Otros problemas también descritos son los secundarios a los recursos materiales $(11,3 \%)$ o los de índole profesional $(10,4 \%)$. Por último, también se mencionan aspectos relacionados con las características del paciente $(9,6 \%)$ o con el medio donde se presta la asistencia.

Los encuestados refieren que el aspecto que más ha mejorado en el control de la infección por el VIH es el avance en los tratamientos farmacológicos (34\%), pero paradójicamente también es el tratamiento antirretroviral, por su toxicidad o por la posibilidad de fracaso, el aspecto de futuro que más preocupa $(31,9 \%)$. Si se pide al profesional que plantee qué mejoraría, el 34,5\% de los encuestados refiere que algunos aspectos profesionales, mientras que el $24,1 \%$ considera que debe mejorar la asistencia especializada y el $11,2 \%$ opina que debe mejorar la asistencia global. Los aspectos, sin embargo, considerados más positivos son los procedimientos de control ya existentes $(25 \%)$, la accesibilidad a los profesionales $(14,9 \%)$ y la proximidad médico-paciente $(14,4 \%)$. Los aspectos más negativos citados son los relacionados con la gestión sanitaria (20\%), los relacionados con el perfil del paciente $(20,9 \%)$ y los secundarios al sistema penitenciario $(20,3 \%)$.

\section{DISCUSIÓN}

El perfil del médico que trabaja hoy en el ámbito penitenciario es el de un profesional mayor de 40 años, médico general o especialista en Medicina de Familia y Comunitaria y con una gran experiencia en el ejercicio profesional en prisión. Prácticamente todos los facultativos atienden pacientes con infección por el VIH. Se observa una relación directa entre el tiempo de ejercicio en prisiones y el tiempo que llevan atendiendo a estos pacientes (a mayor tiempo, mayor probabilidad de atención). Sin embargo, sólo la mitad de los médicos prescriben tratamientos antirretrovirales y muchísimos menos (alrededor del $10 \%)$ prescriben combinaciones de rescate.

Es de destacar también que sólo la mitad de los médicos se consideran formados adecuadamente para atender pacientes infectados por el VIH. No obstante, la mayoría afirma haber hecho formación específica de postgrado en la materia y más del $60 \%$ afirma asistir a más de una reunión científica relacionada con esta patología. Hay también una relación directa entre tiempo de ejercicio profesional en prisiones y la prescripción de tratamientos para otras enfermedades infecciosas muy prevalentes, como la tuberculosis o la infección por el VHC.

En general, los médicos consideran que la calidad de la asistencia que prestan a sus pacientes es buena. Algunos entienden, sin embargo, que para esta función deberían estar más apoyados por médicos de otras disciplinas. Y es de resaltar que todavía hay una proporción importante $(27,6 \%)$ que no tienen acceso a pruebas básicas como los tests de resistencias a fármacos antirretrovirales. La mala adherencia al TARV y la comorbilidad con otros procesos, son percibidas como los principales problemas de la infección por el VIH en nuestro medio. Respecto al futuro, lo que más preocupa es lo derivado de la cronificación de la enfermedad, y en concreto, todo lo relacionado con el TARV a largo plazo: resistencias, efectos adversos, interacciones... Finalmente, debe resaltarse que el ambiente penitenciario se percibe como un importante factor que dificulta la atención sanitaria, de estos y otros pacientes, ya que es considerado un medio paralelo a la red sanitaria normaliza$\mathrm{da}$, en el que no se prioriza la salud y que puede ocasionar el aislamiento del profesional de la salud.

\section{CORRESPONDENCIA}

\author{
Dr. Pablo Saiz de la Hoya Zamácola \\ Centro penitenciario Fontcalent \\ Servicios Médicos \\ Polígono Pla de la Vallonga, sn \\ 03113 Alicante
}

Tel: 965287355 ext: 173

e-mail: Pablos@coma.es

\section{REFERENCIAS BIBLIOGRÁFICAS}

1. Gamella JF, Alvárez-Roldán A. Drogas de Síntesis en España. Patrones y tendencias de adquisi- 
ción y consumo. Madrid: Ministerio del Interior, Delegación del Gobierno para el Plan Nacional sobre Drogas, 1997

2. Caylà JA, Marco A, Bedoya A, Guerrero R, García J, Martín V, et al. Differential Characteristics of AIDS Patients with a history of Prison Internment. Int J Epidemiol 1995; 24: 1188-1196.

3. http://www.mir.es/INSTPEN/INSTPENI/ Gestion/Estadisticas_mensuales/2008/06/

4. Anónimo. Barcelona: Boletí penitenciari número 1, 2008.

5. Marco A. Módulo 3: Problemática psicosocial en VIH: especificidad del paciente ingresado en prisión. En: Programa Psicosocial para prisiones. Madrid: SCM, 2004.

6. Gamella JF, Álvarez-Roldán A. Drogas de Síntesis en España. Patrones y tendencias de adquisición y consumo. Madrid: Ministerio del Interior, Delegación del Gobierno para el Plan Nacional sobre Drogas, 1997

7. Zunzunegui MV, Rodríguez MA, Sarasqueta C. Drogadicción intravenosa y riesgo de infección por VIH en Madrid, 1990. Gac Sanit 1993; 7: 2-11.
8. Cayla JA, Marco A, Bedoya A, Guerrero RA, García J, Martín V, et al. Differential Characteristics of AIDS Patients with a history of Prison Internment. Int J Epidemiol 1995; 24: 1188-96.

9. Martín V, Bayas JM, Laliga A, Pumarola T, Vidal J, Jiménez de Anta MT, et al. Seroepidemiology of HIV-1 infection in a Catalonian penitentiary. AIDS. 1990 Oct; 4 (10): 1023-6.

10. Saiz de la Hoya P, Bedia M, Murcia J, Cebriá J, Sánchez-Payá J, Portilla J. Factores predictivos de infección por el VIH, VHC y coinfección en la población reclusa de una prisión española. Enferm Infecc Microbiol Clin. 2005; 23:53-7.

11. Boletín Epidemiológico de Instituciones Penitenciarias. 2003; 8 (1): 1-2.

12. Saiz de la Hoya P, Ortega E, Alia C, Aranda MJ, Asensi V, García-Guerrero J, et al. Protocolos de coordinación entre servicios sanitarios penitenciarios y hospitales de referencia. Infección VIH y VHC. Madrid: SCM, 2004.

13. Marco A . Manual de Formación: Adherencia al tratamiento con antiretrovirales dentro y fuera de prisión. Doyma eds. Madrid: 1999. 